\title{
Phospholipid-Dependent Functions of a Macrocyclic Analogue of the Ion- Channel-Forming Antibiotic Gramicidin A
}

\author{
Ji Mao, ${ }^{a}$ Hiroaki Itoh, ${ }^{a}$ Kaori Sakurai, ${ }^{b}$ and Masayuki Inoue ${ }^{*, a}$ \\ ${ }^{a}$ Graduate School of Pharmaceutical Sciences, The University of Tokyo; 7-3-1 Hongo, Bunkyo-ku, Tokyo 113-0033, \\ Japan: and ${ }^{b}$ Department of Biotechnology and Life Science, Tokyo University of Agriculture and Technology; 2-24- \\ 16 Naka-cho, Koganei, Tokyo 184-8588, Japan.
}

Received November 1, 2019; accepted November 28, 2019

An ion-channel-forming natural peptide, gramicidin A (1), exhibits potent antimicrobial activity against Gram-positive bacteria, although medical applications are limited to topical use due to its mammalian cytotoxicity. We recently reported that the artificial macrocyclic analogue 2 provides a promising starting point for developing new ion-channel-based systemic antibacterial agents because of its low mammalian cytotoxicity compared to that of the parent 1 . To dissect the molecular factors involved in the species selectivity of 2, we evaluated the ion transport activities, phospholipid affinities, and conformational properties of 1 and 2 using various compositions of phospholipids. A combination of lipid dot blot assays and circular dichroism (CD) analysis with $\mathrm{H}^{+} / \mathrm{Na}^{+}$exchange assays revealed that the higher $\mathrm{H}^{+} / \mathrm{Na}^{+}$exchange activity of 2 than that of 1 in liposomes containing 1-palmitoyl-2-oleoyl-sn-glycero-3-phosphatidylcholine (POPC) or 1-palmitoyl-2-oleoyl-sn-glycero-3-phospho-(1'-rac-glycerol) (POPG) is attributable to its higher affinity towards the phospholipids than that of 1 . Notably, we also discovered that 2 showed weaker $\mathrm{H}^{+} / \mathrm{Na}^{+}$exchange activity in liposomes containing 1-palmitoyl-2-oleoyl-sn-glycero-3-phosphatidylethanolamine (POPE). CD analysis of 2 in liposomes indicated that the weak $\mathrm{H}^{+} / \mathrm{Na}^{+}$exchange activity is induced by disturbance of the ion-conducting $\beta^{6.3}$-helical conformation in the POPE-containing lipid bilayer. These results suggest that the POPE-induced attenuation of the ion-conducting activity of 2 contributes to the alleviation of undesirable mammalian cytotoxicity of 2 compared to that of 1 .

Key words antibiotic; ion channel; natural product; peptide; macrocycle

\section{Introduction}

The emergence and spread of multidrug-resistant bacteria are global issues affecting human health. ${ }^{1-3)}$ To maintain the efficacy of chemotherapy for life-threatening bacterial infections, the discovery and development of promising antimicrobial lead compounds have been of great importance. ${ }^{4,5)}$ In this context, membrane-targeting antibiotics are an attractive class of compounds because they are fundamentally less likely to induce drug resistance than antibiotics that target other bacterial biopolymers due to the relatively conserved molecular composition of lipids. ${ }^{6,7)}$ However, most membrane-targeting compounds cannot recognize species-specific components within the phospholipid bilayer. Synthetic alteration of a known membrane-targeting compound would be a potentially effective strategy to improve its species selectivity and thereby to reduce undesirable mammalian cytotoxicity. Nonetheless, this approach is highly challenging due to the unknown chemical basis for designing such modulated analogues based on the structure of the parent molecule.

Gramicidin A (1, molecular weight $=1882 \mathrm{Da}$, Fig. 1) is a naturally occurring membrane-targeting antibacterial peptide. The linear D,L-alternating 15-amino-acid sequence of $\mathbf{1}$, which was discovered in the soil bacterium Bacillus brevis in $1939,{ }^{8-10)}$ exhibits potent antimicrobial activity against Grampositive bacteria. The mode of action of $\mathbf{1}$ has been explained by a loss in membrane potential by forming an ion channel. ${ }^{11)}$ In a lipid bilayer, the D,L-alternating 15 amino acid residues of 1 form a head-to-head dimer consisting of two $\beta$-stranded helical structures with 6.3 residues per turn $\left(\beta^{6.3}\right.$-helix). ${ }^{12-16)}$
The $\beta^{6.3}$-helix dimer is known to function as a transmembrane channel, which conducts monovalent cations (e.g., $\mathrm{H}^{+}, \mathrm{Na}^{+}$, and $\mathrm{K}^{+}$) through hydrophilic pores. ${ }^{17)}$ The ion transport caused by a channel of $\mathbf{1}$ is believed to abolish the membrane potential, leading to bacterial growth inhibition and death. The antibacterial activity of $\mathbf{1}$ is potentially useful for developing new membrane-targeting antibacterial agents for treating drug-resistant bacterial infection. Despite the high potential of $\mathbf{1}$ as an antibacterial agent, the clinical application of $\mathbf{1}$ has been limited to topical use because of its strong mammalian cytotoxicity, which is likely due to the lack of species selectivity for ion-channel formation. ${ }^{18)}$

To fully harness the bioactivity of $\mathbf{1}$ for broader medical applications, species selectivity in channel formation by 1 must be achieved to reduce toxicity to mammalian cells. Our recent synthetic efforts to develop new gramicidin analogues led to a serendipitous discovery of a promising macrocycle $\mathbf{2}^{19)}$ (Fig. 1). Analogue 2 displayed lower hemolytic activity $(<10 \%$ for 2 and $100 \%$ for $\mathbf{1})$ and mammalian cytotoxicity $\left(\mathrm{IC}_{50}\right.$ against P388 mouse leukemia cells $=2.8 \mu \mathrm{M}$ for 2 and $0.0032 \mu \mathrm{M}$ for 1) than the parent natural product 1 while maintaining similar antibacterial activity against several Gram-positive strains (e.g., minimum inhibitory concentration (MIC) against Staphylococcus aureus $=3.3 \mu \mathrm{M}$ for 2 and 1). ${ }^{20-28)}$ We initially designed 2 to stabilize the biologically active $\beta^{6.3}$-helical conformation by forming a 27 -membered macrocycle consisting of a sidechain-sidechain bridging structure between residues-4 and -10 (6 residues apart in the sequence). Understanding the molecular factors underlying the change in cytotoxicity against 


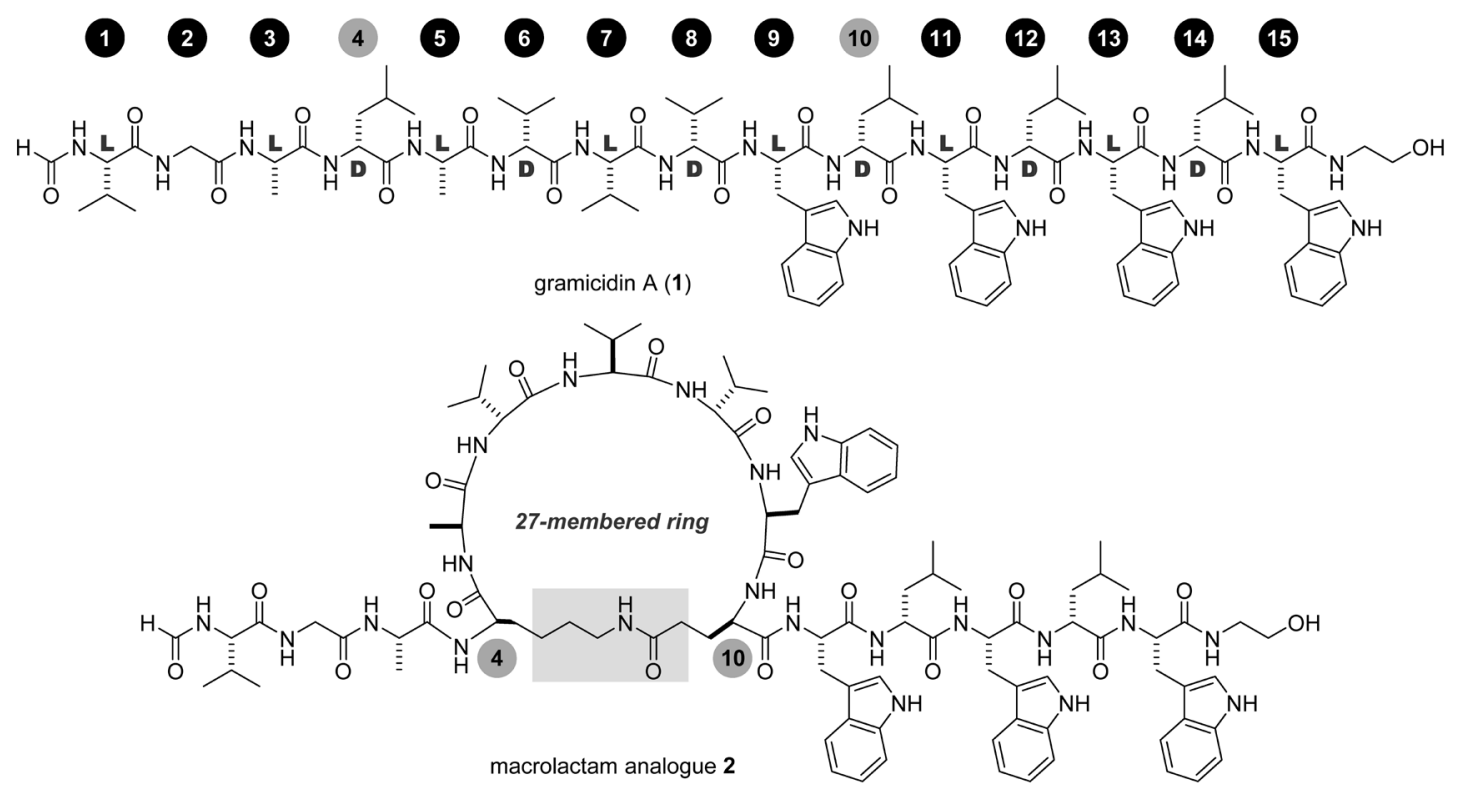

\begin{tabular}{|c|c|c|c|}
\hline compounds & $\begin{array}{c}\text { antimicrobial activity } \\
\text { against } S \text {. aureus } \\
\text { (MIC, } \mu \mathrm{M} \text { ) }\end{array}$ & $\begin{array}{c}\text { hemolysis } \\
(\%)\end{array}$ & $\begin{array}{c}\mathrm{IC}_{50} \\
\text { against } \mathrm{P} 388 \text { cells } \\
(\mu \mathrm{M})\end{array}$ \\
\hline 1 & 3.3 & 100 & 0.0032 \\
\hline 2 & 3.3 & $<10$ & 2.8 \\
\hline
\end{tabular}
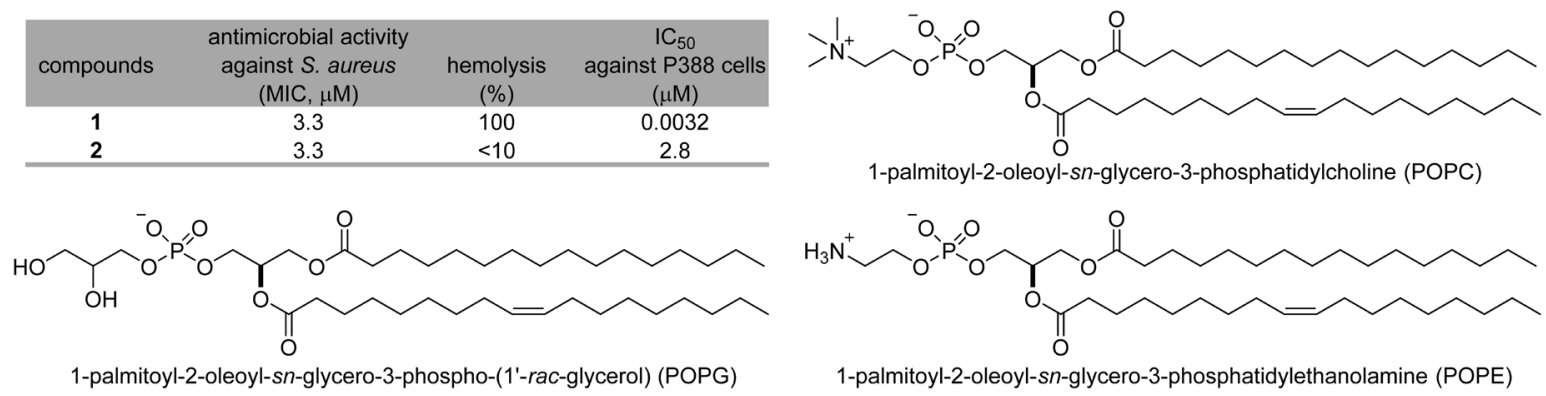

Fig. 1. Structures of Gramicidin A (1) and the Macrolactam Analogue 2

Previously reported biological activities of $\mathbf{1}$ and $\mathbf{2},{ }^{19)}$ and the structures of POPC, POPG, and POPE are also displayed.

P388 cells should allow us to further develop better antibiotics based on 2 . We hypothesized that the difference between the activity of $\mathbf{1}$ and $\mathbf{2}$ may arise from the interactions between the sidechain bridging structure of $\mathbf{2}$ (highlighted in gray, Fig. 1) and specific lipid components of the cell membrane of S. aureus and P388 mouse leukemia cells. To understand the possible basis for the distinct activity of $\mathbf{2}$ in bacterial and mammalian membranes, we analyzed the functions and properties of $\mathbf{1}$ and $\mathbf{2}$ in different compositions of phospholipid membranes in this study.

\section{Results and Discussion}

Experimental Design Non-selective ion conductance has been proposed as a major cause of the cytotoxicity of $\mathbf{1}$. In principle, the ion-conducting activity of $\mathbf{1}$ and $\mathbf{2}$ can be defined by the total activity of multiple channel molecules in each cell membrane. The total ion conductance of $\mathbf{1}$ and $\mathbf{2}$ can be affected by both the number of total channel-forming molecules and the number of those in the active conformation in each cell. We therefore investigated whether these two factors are differentially affected by the surrounding phospholipid compositions in the cases of $\mathbf{1}$ and $\mathbf{2}$ by evaluating their phospholipid affinity, ion transport activity, and conformation in various phospholipids. In a previous study, we assessed the ion transport activity of $\mathbf{1}$ and $\mathbf{2}$ in liposomes consisting of a 4:1 mixture of egg yolk phosphatidylcholine (EYPC) and palmitoyl-2-oleoyl-sn-glycero-3-phosphatidylcholine (POPC) or 1-palmitoyl-2-oleoyl-sn-glycero-3-phospho-(1'-rac-glycerol) (POPG). ${ }^{19)}$ Zwitter ionic POPC was used as a model of a mammalian plasma membrane, ${ }^{29)}$ and anionic POPG was used as a model of a negatively charged Gram-positive bacterial membrane, ${ }^{30)}$ because $\mathrm{PC}$ and $\mathrm{PG}$ make up $40 \%$ of the phospholipids in mammalian cells and $58 \%$ of the total lipids in Staphylococcus aureus, respectively. The ion transport activities of $\mathbf{1}$ and $\mathbf{2}$ were found to be comparable in POPCand POPG-containing membranes, suggesting that these major membrane components do not contribute to different behaviors of $\mathbf{1}$ and $\mathbf{2}$. We thus hypothesized that the mammalian cytotoxicity of $\mathbf{2}$ may be attenuated in the presence of phosphatidylethanolamine, such as 1-palmitoyl-2-oleoyl-snglycero-3-phosphatidylethanolamine (POPE), the second most prevalent component of mammalian plasma membranes $(20 \%$ of phospholipids). ${ }^{29)}$ POPE was therefore used in addition to EYPC, POPE, and POPG for liposome preparation to assess the assumption in this study.

Ion Transport Activity of Gramicidin A and Macrocyclic Analogue in Different Phospholipid Membranes To investigate whether $\mathbf{1}$ and $\mathbf{2}$ show lipid-dependent ion channel activity in the phospholipid bilayer, the $\mathrm{H}^{+} / \mathrm{Na}^{+}$exchange activity of $\mathbf{1}$ and $\mathbf{2}$ in various phospholipids was initially evaluated using liposomes as model membranes. The liposomes were prepared as large unilamellar vesicles (LUVs) consisting of a 4:1 mixture of EYPC and one of the three phospholipids, POPE, POPC, or POPG. Briefly, a $\mathrm{pH}$-dependent 
a

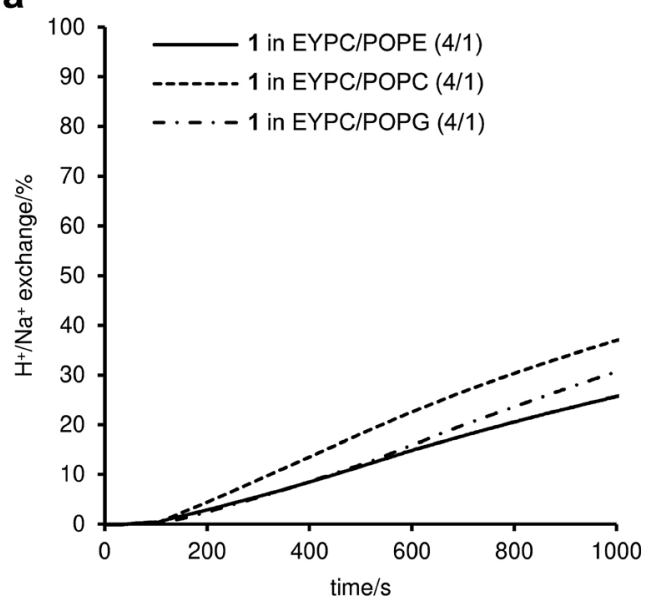

b

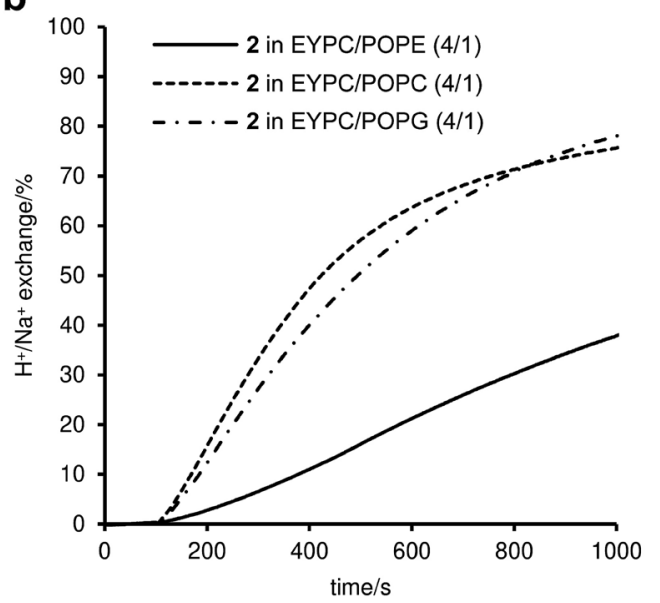

Fig. 2. Time Course of Ion Transport

(a) $\mathrm{H}^{+} / \mathrm{Na}^{+}$exchange caused by 1 and (b) $2 \cdot \mathrm{H}^{+} / \mathrm{Na}^{+}$exchange across the lipid bilayers of LUVs with a $\mathrm{pH}$-gradient was evaluated. Data were recorded at a peptide concentration of $100 \mathrm{nM}$. The results for EYPC/POPC (4/1) and EYPC/POPG (4/1) were previously reported. ${ }^{19)}$

fluorescent dye, trisodium 8-hydroxypyrene-1,3,6-trisulfonate (pyranine), ${ }^{31,32)}$ was encapsulated in the LUVs. Exchanging the external buffer allows a $\mathrm{pH}$ gradient to be generated across the LUV membrane. By forming cation-conducting channels in the lipid bilayers, $\mathrm{H}^{+}$transport occurred according to the $\mathrm{pH}$ gradient along with the counter transport of $\mathrm{Na}^{+}$, which can be detected by changes in the fluorescent intensity of pyranine. As shown in Fig. $2 \mathrm{a}$, the $\mathrm{H}^{+} / \mathrm{Na}^{+}$exchange activity of the parent natural product $\mathbf{1}$ was similar in the three kinds of LUV that differed in one of the phospholipid components $(26 \%$ in EYPC/POPE, 37\% in EYPC/POPC, and 31\% in EYPC/POPG at $1000 \mathrm{~s})$. In contrast, analogue 2 displayed significantly reduced activity in the LUVs consisting of EYPC/POPE (38\% at $1000 \mathrm{~s}$ ) compared to those in the LUVs consisting of EYPC/ POPC $(76 \%$ at $1000 \mathrm{~s})$ and $\mathrm{EYPC} / \mathrm{POPG}(78 \%$ at $1000 \mathrm{~s})$ that were analyzed in our previous study ${ }^{19)}$ (Fig. 2b). The activity of 2 in EYPC/POPC and EYPC/POPG was much higher than that of 1, while its activity in EYPC/POPE was at a similar level to that of $\mathbf{1}$ in the three different LUVs. This result indicated that in a simple membrane model, analogue $\mathbf{2}$ exerts more potent ion transport activity than $\mathbf{1}$ and the presence of POPE disrupts this trend. Although 1 showed weaker activity in EYPC/POPE than in EYPC/POPC and EYPC/POPG, the degree of effect was substantially less than that observed for a

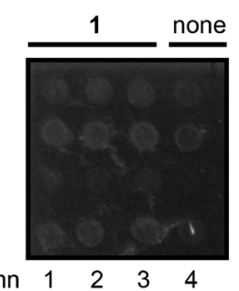

b

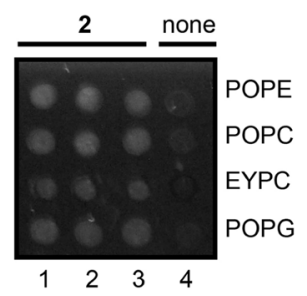

C

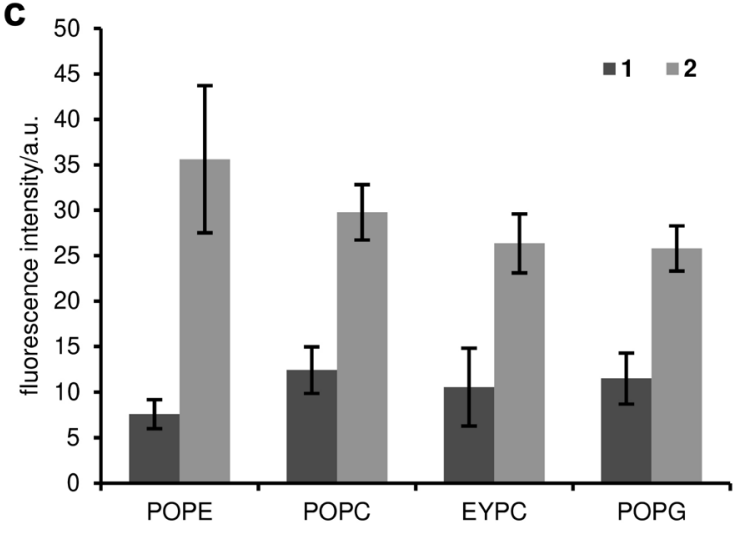

Fig. 3. Lipid Dot Blot Assay

(a) Gray-scale image of the fluorescence from $\mathbf{1}$ and (b) $\mathbf{2}$ absorbed on the PVDF membrane sheet after $2 \mathrm{~h}$ of incubation. Columns $1-3$ show spots of the peptide $(0.1 \mathrm{nmol})$ with the phospholipids $(10 \mathrm{nmol})$, and column 4 shows control spots (phospholipids without the peptide). (c) Fluorescence intensities of 1 and 2 . The background fluorescence of each control spot was subtracted from the fluorescence of $\mathbf{1}$ or 2. Data are plotted as the mean \pm standard deviation (S.D.).

2. These results suggested that the bulk ion transport activity of $\mathbf{2}$ may be selectively attenuated in a membrane environment containing PE.

Phospholipid Affinity of Gramicidin A and Macrocyclic Analogue The total ion transport mediated by $\mathbf{1}$ and $\mathbf{2}$ should be correlated with the number of molecules partitioned in the membrane. We next evaluated the phospholipid affinities of $\mathbf{1}$ and $\mathbf{2}$ by lipid dot blot analysis to assess the membrane partitioning efficiency of the peptides. ${ }^{33)}$ In this assay, phospholipids were spotted and absorbed on the surface of a polyvinylidenfluoride (PVDF) membrane with hydrophobic interactions. Then, a peptide solution was added to the lipid spot on the sheet, and the excess peptide was removed from the membrane by washing. The phospholipid affinity of the peptide was evaluated by measuring the fluorescence of each spot emitted from the four tryptophan residues of $\mathbf{1}$ and $\mathbf{2}$ by UV irradiation (Figs. 3a and 3b). Image analysis revealed that $\mathbf{2}$ showed higher fluorescence intensity than $\mathbf{1}$ (Fig. 3c), which suggested that $\mathbf{2}$ generally has higher affinity to phospholipids than 1. Furthermore, the affinities of $\mathbf{1}$ and $\mathbf{2}$ towards different phospholipids were assessed. The mean fluorescent intensity of $\mathbf{1}$ on the POPE spot was smaller than those of $\mathbf{1}$ on other phospholipid spots, and the mean fluorescent intensity of $\mathbf{2}$ on the POPE spot was higher than those of $\mathbf{2}$ on other phospholipid spots, although the differences in the fluorescent intensities of $\mathbf{1}$ and $\mathbf{2}$ in the tested phospholipids were not statistically significant. The data suggested that 2 binds the four different phospholipids similarly and does not discriminate among them in terms of binding affinity.

POPE-Induced Destabilization of the $\beta^{6.3}$-Helical Structure of the Macrocyclic Analogue To assess the lipid-dependent conformational properties of $\mathbf{1}$ and $\mathbf{2}$ in the phospho- 
lipid bilayer, circular dichroism (CD) analysis was carried out using various compositions of liposomes. In the LUVs consisting of EYPC/POPE (4/1), EYPC/POPC (4/1), and EYPC/ POPG (4/1), the parent natural product 1 provides two positive peaks at approximately 220 and $240 \mathrm{~nm}$, which are derived from the ion-conducting $\beta^{6.3}$-helical conformation ${ }^{34-36)}$ (Fig. 4). Analogue 2 also exhibited similar peaks, which indicated that 2 may also form a $\beta^{6.3}$-helix. The CD spectrum of $\mathbf{1}$ in EYPC/POPE showed slightly enhanced $\beta^{6.3}$-helix signals compared to those observed in EYPC/POPC and EYPC/POPG. Analogue 2 showed very weak signals in EYPC/POPE compared with EYPC/POPC and EYPC/POPG (Fig. 4b). These results suggested that 2 cannot efficiently form a $\beta^{6.3}$-helix and does not have a well-defined secondary structure in the presence of POPE. The structural basis for this selective destabilization of the $\beta^{6.3}$-helix conformation in $\mathbf{2}$ by POPE is currently not known. However, assuming that the $\beta^{6.3}$-helix conformation is the active conformation of $\mathbf{2}$ for ion transport, we surmise that the formation of ion-conducting channels by $\mathbf{2}$ is disfavored in mammalian cell membranes that contain PE as the second most prevalent lipid component, as opposed to S. aureus membranes, which do not have PE. ${ }^{30}$

\section{Conclusion}

In conclusion, we revealed the phospholipid dependence of the conformational and functional properties of the macrocyclic gramicidin A analogue $\mathbf{2}$ as a possible contributing factor to the selective cytotoxicity of $\mathbf{2}$ in bacterial over mammalian cells. First, the natural product $\mathbf{1}$ possesses nonselective $\mathrm{H}^{+} / \mathrm{Na}^{+}$exchange activity in LUVs composed of EYPC/POPC, EYPC/POPG or EYPC/POPE, whereas analogue 2 displays reduced activity in $\mathrm{EYPC/POPE}$ compared to that in $\mathrm{EYPC} /$ POPC or EYPC/POPG. Lipid dot blot analysis indicated that this weak ion exchange function of $\mathbf{2}$ in EYPC/POPE is not due to the lower affinity of $\mathbf{2}$ towards POPE than POPC or POPG. According to CD analysis, the attenuation of $\mathrm{H}^{+} / \mathrm{Na}^{+}$ exchange by 2 in EYPC/POPE may be explained by a scenario where the formation of the ion-conducting $\beta^{6.3}$-helical conformation is disfavored in lipid membranes containing PE. Since PE is one of the major lipid components in mammalian cell membranes and not in $S$. aureus membranes, the $\beta^{6.3}$-helix content of analogue $\mathbf{2}$ may be low in mammalian membranes, which may contribute to the reduced toxicity towards mammalian cells compared with that of the natural product 1 . How $\mathrm{PE}$ disturbs the $\beta^{6.3}$-helical formation in $\mathbf{2}$ and how efficiently it does so are of great interest, which warrants a structural investigation on the interaction between 2 and PE. Second, analogue 2 exhibits more potent $\mathrm{H}^{+} / \mathrm{Na}^{+}$exchange activity than the parent natural product $\mathbf{1}$ in the LUVs consisting of EYPC/ POPC or EYPC/POPG, which may be explained by its higher binding affinity to phospholipids than that of $\mathbf{1}$. However, the different ion transport activity observed for $\mathbf{1}$ and $\mathbf{2}$ in the LUVs does not directly correlate with the fact that $\mathbf{1}$ and $\mathbf{2}$ show similar antimicrobial activities against $S$. aureus, which suggested that other additional factors are likely involved. Further mechanistic analysis on the functional and conformational perturbations of $\mathbf{2}$ in specific phospholipid bilayers would provide a design principle for the attenuation of the mammalian cytotoxicity of ion-channel-forming compounds, leading to the development of ion-channel-based systemic antibacterial agents.

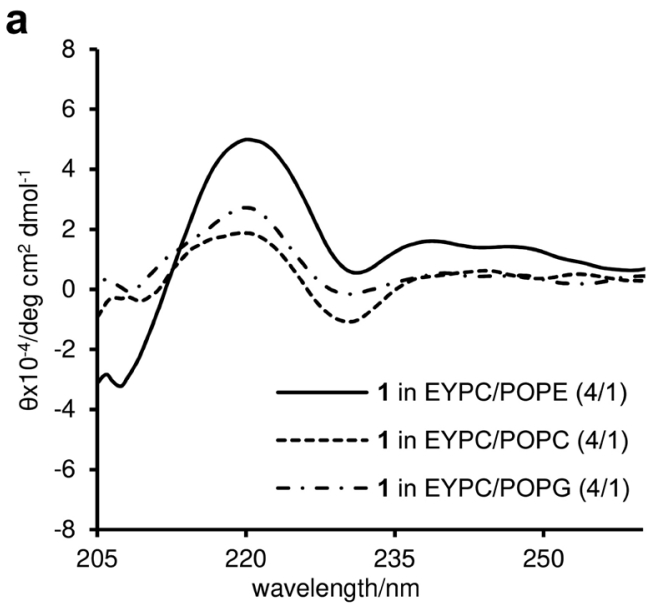

b

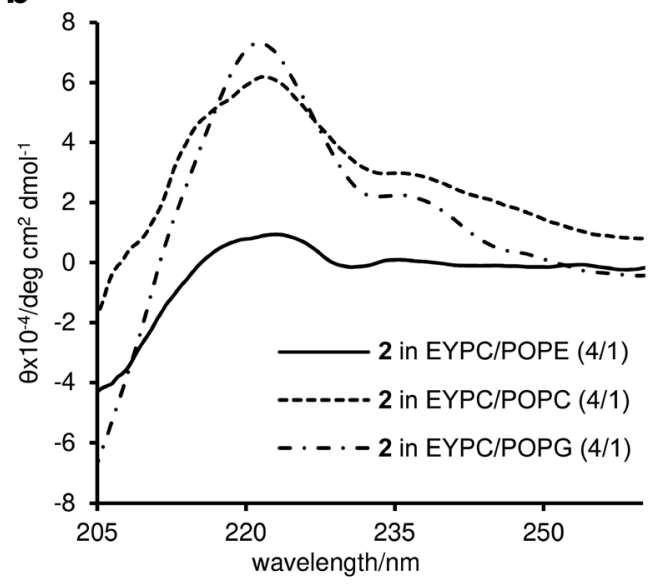

Fig. 4. CD Analysis of $\mathbf{1}$ and $\mathbf{2}$ in LUVs

(a) CD spectra of 1 in LUVs consisting of EYPC/POPE (4/1), EYPC/POPC (4/1) or EYPC/POPG (4/1). The results for EYPC/POPC (4/1) and EYPC/POPG (4/1) were previously reported. ${ }^{19)}$ (b) CD spectra of 2 in LUVs consisting of EYPC/POPE $(4 / 1)$, EYPC/POPC $(4 / 1)$ or EYPC/POPG (4/1). Data were recorded at a peptide concentration of $20 \mu \mathrm{M}$. The results for EYPC/POPC (4/1) and EYPC/POPG (4/1) were previously reported. ${ }^{19)}$

\section{Experimental}

Ion Transport Assay Pyranine-encapsulated LUVs were prepared according to the thin-film hydration method, followed by extrusion through Nuclepore polycarbonate filters (Whatman, United Kingdom) mounted in the mini-extruder apparatus (Avanti Polar Lipids, U.S.A.). A solution of EYPC $(13.6 \mathrm{mg}, 17.9 \mu \mathrm{mol})$ and POPE, POPC or POPG $(4.47 \mu \mathrm{mol})$ in $\mathrm{CHCl}_{3} / \mathrm{MeOH}(3 / 1)$ was evaporated, and dried under vacuum for $1 \mathrm{~h}$ to form a lipid thin film. The thin film was hydrated and suspended in $1.50 \mathrm{~mL}$ of pyranine-containing $\mathrm{pH} 5.5$ phosphate buffer solution $(0.4 \mathrm{M}$ sodium phosphate, $1 \mathrm{mM}$ pyranine) by sonication. After three-times freeze-thaw cycles, the lipid suspension was extruded 19 times through a polycarbonate filter with $0.1 \mu \mathrm{m}$ of pore size in the diameter. The external pyranine-containing buffer was replaced by pyranine-free $\mathrm{pH} 5.5$ phosphate buffer solution $(0.4 \mathrm{M}$ sodium phosphate) through size exclusion chromatography using a disposable PD-10 column (GE Healthcare, U.S.A.). Lipid concentration of the resultant pyranine-encapsulated LUV suspension was deduced from the phosphatidylcholine concentration determined by using Phospholipid C-Test Wako (FUJIFILM Wako Pure Chemical Corporation, Japan). The lipid concentration of the LUV suspension was adjusted to 
$100 \mu \mathrm{M}$ with the pyranine-free $\mathrm{pH} 7.5$ phosphate buffer solution $\left(0.1 \mathrm{M}\right.$ sodium phosphate buffer, $\left.0.3 \mathrm{M} \mathrm{Na}_{2} \mathrm{SO}_{4}\right)$ to generate $\mathrm{pH}$-gradient. A suspension of the LUVs was immediately used for the $\mathrm{H}^{+} / \mathrm{Na}^{+}$transport assay.

A suspension of pyranine-encapsulated $\mathrm{pH}$-gradient LUVs $(790 \mu \mathrm{L})$ was placed in a micro quartz cuvette. The suspension was stirred gently with a magnetic stirring bar at $26^{\circ} \mathrm{C}$ in a FP-6500 spectrofluorometer with EHC-573 Peltier thermostatted cell holder (JASCO, Japan). The fluorescence emission was measured at $520 \mathrm{~nm}$ with the excitation at $460 \mathrm{~nm}$. After equilibration at $26^{\circ} \mathrm{C}$ for $10 \mathrm{~min}$, the fluorescence emission was started to be recorded. After $60 \mathrm{~s}$, a solution of $8 \mu \mathrm{M}$ peptide in dimethyl sulfoxide (DMSO) $/ \mathrm{H}_{2} \mathrm{O}(1 / 4,10.0 \mu \mathrm{L})$ was added via an injection port to the stirring LUV suspension. The fluorescence emission was recorded for $1 \mathrm{~h}$ at a sampling rate of $1 \mathrm{~s}$, and then remaining LUVs were completely lysed by addition of Triton X-100/ $\mathrm{H}_{2} \mathrm{O}(1 / 9,10.0 \mu \mathrm{L})$. The background drift from DMSO addition was cancelled by subtracting the control trace obtained from $\mathrm{DMSO} / \mathrm{H}_{2} \mathrm{O}(1 / 4)$ without peptides. The data were smoothed by moving average. The $\mathrm{H}^{+} / \mathrm{Na}^{+}$transport activities of tested peptides $(I)$ were normalized against $100 \%$ lysis by Triton $\mathrm{X}-100 / \mathrm{H}_{2} \mathrm{O}\left(1 / 9, I_{\max }\right)$ and $0 \%$ lysis $\left(I_{0}\right)$ as following, where $I_{t}$ is the fluorescence intensity at time $t$, and $I_{0}$ is the fluorescence intensity before the addition of peptides (Equation 1).

$$
I=100 \times\left(I_{t}-I_{0}\right) /\left(I_{\max }-I_{0}\right)
$$

Lipid Dot Blot Assay A solution of phospholipid in $\mathrm{CHCl}_{3}$ or $\mathrm{CHCl}_{3} / \mathrm{MeOH}$ (3/1) was spotted twice on the PVDF membrane sheet (Immobilon, $0.2 \mu \mathrm{m}$, Millipore, U.S.A.) using a capillary pipette (minicaps, Hirschmann-Laborgeräte, Germany). The lipid spots on the membrane sheet were dried in the dark overnight. The membrane sheet was placed onto a wetted filter paper for hydration of the lipids. A solution of peptide in buffer $(2 \mu \mathrm{L}, 50 \mathrm{mM}$ in $\mathrm{pH} 7.4$ phosphate buffered saline) was added onto each lipid spot. The membrane sheet was covered and kept in the dark at room temperature for $2 \mathrm{~h}$. The membrane sheet was washed with $\mathrm{pH} 7.4$ phosphate buffered saline for $10 \mathrm{~min}$. The sheet was then placed in the dark box, and UV light ( $254 \mathrm{~nm}$, UVGL-25 compact UV lamp, Upland) was irradiated on the membrane sheet. The resultant sheet was photographed (IXY digital 920 IS, Canon, Japan). The intensity of the fluorescence signals was analyzed by ImageJ. ${ }^{37)}$

CD Measurements A solution of EYPC $(9.12 \mathrm{mg}$, $12.0 \mu \mathrm{mol})$ and POPE, POPC, or POPG $(3.00 \mu \mathrm{mol})$ in $\mathrm{CHCl}_{3} /$ $\mathrm{MeOH}(3 / 1)$ was evaporated, and dried under vacuum for $1 \mathrm{~h}$ to form a lipid thin film. The thin film was hydrated and suspended in $\mathrm{pH} 7.5$ phosphate buffer solution $(1.00 \mathrm{~mL}, 25 \mathrm{mM}$ sodium phosphate, $75 \mathrm{mM} \mathrm{Na}_{2} \mathrm{SO}_{4}$ ) by sonication. After threetimes freeze-thaw cycles, the lipid suspension was extruded 19 times through a polycarbonate filter with $0.1 \mu \mathrm{m}$ of pore size in the diameter. The resultant suspension was purified through size exclusion chromatography using a disposable PD-10 column with by the $\mathrm{pH} 7.5$ phosphate buffer solution. Lipid concentration of the resultant LUV suspension was deduced from the phosphatidylcholine concentration determined by using Phospholipid C-Test Wako. The lipid concentration of the LUV suspension was adjusted to $1 \mathrm{mM}$ with the $\mathrm{pH} 7.5$ phosphate buffer solution. A suspension of the LUVs was im- mediately used for the CD measurements.

To a solution of each peptide $(8.00 \mathrm{nmol})$ in $\mathrm{MeOH}(10.0 \mu \mathrm{L})$ was added a suspension of LUVs $(390 \mu \mathrm{L})$. CD spectra were recorded in a $0.2 \mathrm{~cm}$ pathlength cuvette at $25^{\circ} \mathrm{C}$ using a $\mathrm{J}-820$ spectropolarimeter with Peltier thermostatted cell holder (JASCO). Data were acquired for $200-280 \mathrm{~nm}$ at every $0.5 \mathrm{~nm}$ with a standard sensitivity mode (100 mdeg) applying $2 \mathrm{~nm}$ band width. Each measurement was repeated four times at a scanning speed of $100 \mathrm{~nm} / \mathrm{min}$ with a response time of $2 \mathrm{~s}$ and at $25^{\circ} \mathrm{C}$. In all cases, the peptide-free lipid suspension spectra were subtracted from the peptide-lipid suspension spectra. Intensity of $\mathrm{CD}$ spectra were expressed in units of molar ellipticity (deg $\left.\mathrm{cm}^{2} \mathrm{dmol}^{-1}\right)$ and plotted against the wavelength (nm).

Acknowledgments This research was financially supported by Grants-in-Aid for Scientific Research (S) (JSPS, JP17H06110) to M.I. and for Early-Career Scientists (JSPS, JP19K15704) to H.I. A JSPS Fellowship to J.M. is gratefully acknowledged.

Conflict of Interest The authors declare no conflict of interest.

\section{References}

1) Walsh C., Wencewicz T., "Antibiotics: Challenges, Mechanisms, Opportunities," ASM Press, Washington, DC, 2016.

2) Brown E. D., Wright G. D., Nature (London), 529, 336-343 (2016). 3) Blair J. M. A., Webber M. A., Baylay A. J., Ogbolu D. O., Piddock L. J. V., Nat. Rev. Microbiol., 13, 42-51 (2015).

4) Chellat M. F., Raguž L., Riedl R., Angew. Chem. Int. Ed., 55, 6600-6626 (2016).

5) O'Connell K. M. G., Hodgkinson J. T., Sore H. F., Welch M., Salmond G. P. C., Spring D. R., Angew. Chem. Int. Ed., 52, 1070610733 (2013)

6) Steinbuch K. B., Fridman M., Med. Chem. Commun., 7, 86-102 (2016).

7) Epand R. M., Walker C., Epand R. F., Magarvey N. A., Biochim. Biophys. Acta, 1858, 980-987 (2016).

8) Sarges R., Witkop B., J. Am. Chem. Soc., 87, 2011-2020 (1965).

9) Hotchkiss R. D., Dubos R. J., J. Biol. Chem., 132, $791-792$ (1940).

10) Dubos R. J., J. Exp. Med., 70, 1-10 (1939).

11) Kelkar D. A., Chattopadhyay A., Biochim. Biophys. Acta, 1768, 2011-2025 (2007).

12) Kovacs F., Quine J., Cross T. A., Proc. Natl. Acad. Sci. U.S.A., 96, 7910-7915 (1999).

13) Wallace B. A., J. Struct. Biol., 121, 123-141 (1998).

14) Ketchem R. R., Hu W., Cross T. A., Science, 261, 1457-1460 (1993). 15) Arseniev A. S., Barsukov I. L., Bystrov V. F., Lomize A. L., Ovchinnikov Y. A., FEBS Lett., 186, 168-174 (1985).

16) Urry D. W., Goodall M. C., Glickson J. D., Mayers D. F., Proc. Natl. Acad. Sci. U.S.A., 68, 1907-1911 (1971).

17) Myers V. B., Haydon D. A., Biochim. Biophys. Acta, 274, 313-322 (1972).

18) Knopik-Skrocka A., Bielawski J., Cell. Mol. Biol. Lett., 7, 31-48 (2002).

19) Mao J., Kuranaga T., Hamamoto H., Sekimizu K., Inoue M., ChemMedChem, 10, 540-545 (2015).

20) For examples of chemically modified 1 analogues, see refs 20-25: Wang F., Qin L., Pace C. J., Wong P., Malonis R., Gao J., ChemBioChem, 13, 51-55 (2012).

21) Macrae M. X., Blake S., Mayer M., Yang J., J. Am. Chem. Soc., 132, 1766-1767 (2010).

Reiß P., Al-Momani L., Koert U., ChemBioChem, 9, 377-379 
(2008).

23) Pfeifer J. R., Reiß P., Koert U., Angew. Chem. Int. Ed., 45, 501-504 (2006).

24) Woolley G. A., Jaikaran A. S. I., Zhang Z., Peng S., J. Am. Chem. Soc., 117, 4448-4454 (1995).

25) Stankovic C. J., Heinemann S. H., Delfino J. M., Sigworth F. J., Schreiber S. L., Science, 244, 813-817 (1989).

26) For reviews of chemically modified 1 analogues, see refs. 26-28: Reiß P., Koert U., Acc. Chem. Res., 46, 2773-2780 (2013).

27) Mayer M., Yang J., Acc. Chem. Res., 46, 2998-3008 (2013).

28) Koeppe R. E. II, Andersen O. S., Annu. Rev. Biophys. Biomol. Struct., 25, 231-258 (1996).

29) van Meer G., Voelker D. R., Feigenson G. W., Nat. Rev. Mol. Cell Biol., 9, 112-124 (2008).

30) Epand R. M., Epand R. F., Mol. Biosyst., 5, 580-587 (2009).
31) Clement N. R., Gould J. M., Biochemistry, 20, 1534-1538 (1981)

32) Itoh H., Matsuoka S., Kreir M., Inoue M., J. Am. Chem. Soc., 134, 14011-14018 (2012)

33) Munnik T., Wierzchowiecka M., Methods Mol. Biol., 1009, 253-259 (2013).

34) Sychev S. V., Sukhanov S. V., Barsukov L. I., Ivanov V. T., J. Pept. Sci., 2, 141-156 (1996).

35) Killian J. A., Prasad K. U., Hains D., Urry D. W., Biochemistry, 27, 4848-4855 (1988)

36) Wallace B. A., Veatch W. R., Blout E. R., Biochemistry, 20, 5754 5760 (1981)

37) Rasband W. S., "ImageJ.”: 〈https://imagej.nih.gov/ij/〉, cited 25 October, 2019. 